\title{
Laser-produced plasmas as drivers of laboratory collisionless quasi-parallel shocks
}

\author{
P. V. Heuer, ${ }^{1,}$ a) M. S. Weidl, ${ }^{2}$ R. S. Dorst,${ }^{1}$ D. B. Schaeffer, ${ }^{1,}$ b) S. K. P. Tripathi, ${ }^{1}$ S. Vincena, ${ }^{1}$ C. G. \\ Constantin, ${ }^{1}$ C. Niemann, ${ }^{1, c)}$ and D. Winske ${ }^{3}$ \\ ${ }^{1)}$ Department of Physics and Astronomy, University of California-Los Angeles, Los Angeles, California 90095, \\ USA \\ ${ }^{2)}$ Max-Planck-Institut für Plasmaphysik, Boltzmannstr. 2, 85748 Garching, Germany \\ ${ }^{3)}$ Los Alamos National Laboratory, Los Alamos, New Mexico 87544, USA
}

(Dated: 9 April 2020)

The creation of a repeatable collisionless quasi-parallel shock in the laboratory would provide a valuable platform for experimental studies of space and astrophysical shocks. However, conducting such an experiment presents substantial challenges. Scaling the results of hybrid simulations of quasi-parallel shock formation to the laboratory highlights the experimentally demanding combination of dense, fast, and magnetized background and driver plasmas required. One possible driver for such experiments are high-energy laser-produced plasmas (LPPs). Preliminary experiments at the University of California Los Angeles have explored LPPs as drivers of quasi-parallel shocks by combining the Phoenix Laser Laboratory [Niemann et al. Journal of Instrumentation, 7, 2012] with the Large Plasma Device (LAPD) [Gekelman et al. Review of Scientific Instruments, 87, 2016]. Beam instabilities and waves characteristic of the early stages of shock formation are observed, but spatial dispersion of the laser-produced plasma prematurely terminates the process. This result is illustrated by experimental measurements and Monte-Carlo calculations of LPP density dispersion. The experimentally-validated Monte-Carlo model is then applied to evaluate several possible approaches to mitigating LPP dispersion in future experiments.

\section{INTRODUCTION}

Collisionless shocks in space and astrophysical plasmas are an important source of high-energy particles through the process of diffusive shock acceleration ${ }^{1,2}$. Shocks are discontinuities formed by the interaction of a supersonic inflowing "beam" plasma with a background "core" plasma. These plasmas are often magnetized, so collisionless shocks are classified as either quasi-parallel or quasi-perpendicular based on the shocks are distinct from unmagnetized shocks mediated by the Weibel instability, which require substantial beam anisotropy to self-generate magnetic fields ${ }^{4}$.

In the absence of Coulomb collisions, collisionless shocks couple energy from a super-Alfvénic $\left(v_{b}>v_{A}\right.$ where is the Alfvén velocity) beam plasma to the core plasma through perturbations in the bulk electric and magnetic fields. In the quasi-parallel case coupling is mediated by several electromagnetic ion/ion beam instabilities ${ }^{5,6}$. This coupling proceeds more slowly than in the quasi-perpendicular case, producing a shock region that is correspondingly longer (hundreds of ioninertial lengths instead of approximately one). Large-scale structure is required for diffusive shock acceleration to produce very high energies, so quasi-parallel collisionless shocks can be particularly powerful particle accelerators ${ }^{7}$.

Astrophysical shocks such as supernova remnants have been observed remotely ${ }^{8}$, and spacecraft have made in situ measurements of quasi-parallel planetary and cometary bow

\footnotetext{
${ }^{a)}$ Electronic mail: pheuer@physics.ucla.edu

b) Present address: Department of Astrophysical Sciences, Princeton University, Princeton, New Jersey 08540, USA

c)Electronic mail: cniemann@g.ucla.edu
}

shocks within the solar system ${ }^{9-11}$. However, both remote observations and in-situ spacecraft measurements have inherent limitations as laboratories for understanding shock formation, structure, or particle acceleration. Both rely on natural conditions to vary plasma parameters and as such are inherently irreproducible. Spacecraft also only make measurements along their trajectory, and their motion convolves spatial and temporal variations. All of these limitations could potentially be addressed by laboratory experiments ${ }^{12}$.

A recent series of experiments has been conducted at the University of California Los Angeles (UCLA) to evaluate the potential of laser-produced plasmas (LPPs) as drivers of quasi-parallel collisionless shocks. A beam plasma created using one of two high-energy lasers interacts with a magnetized core plasma produced by the Large Plasma Device (LAPD $)^{15}$. Experiments have observed ion/ion beam instability growth consistent with the very early stages of shock formation. However, spatial dispersion quickly reduces the LPP density below the shock formation threshold, terminating the process.

In Section II of this paper we discuss the theoretical design space for quasi-parallel shock formation experiments. Section III outlines the beam instabilities responsible for quasiparallel shock formation and addresses several theoretical challenges to the direct comparison of laboratory measurements of these instabilities to spacecraft observations. Section IV reports the experimental observation of beam instabilities at UCLA that represent the early stages of quasi-parallel shock formation and demonstrates that the process is terminated prematurely by spatial dispersion of the LPP. Section V illustrates this density dispersion using a 3D Monte-Carlo calculation and evaluates a possible solution to this problem using multiple laser pulses. Finally, our conclusions are summarized in Section VI. 


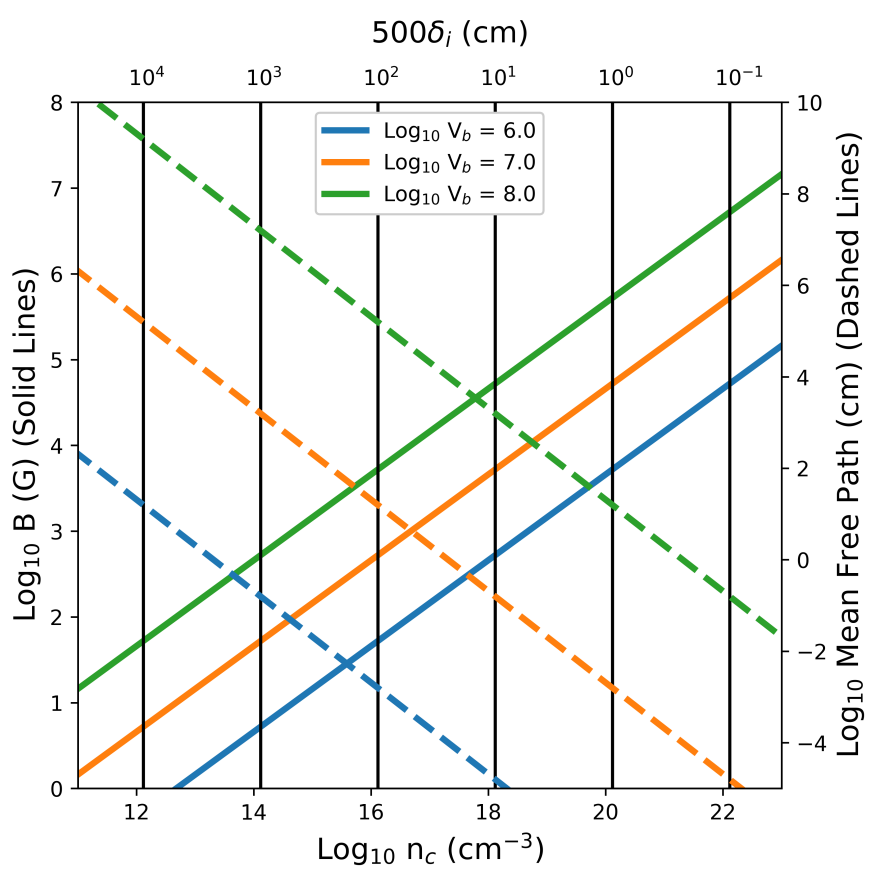

FIG. 1. Required background magnetic field (solid lines) to maintain $M_{A}=10$ for different beam velocities ( $v_{b}$ in $\mathrm{cm} / \mathrm{s}$, line colors) as a function of background density $\left(n_{c}\right.$ in $\left.\mathrm{cm}^{-3}\right)$ calculated for proton beam and core plasmas. The beam-ion/core-ion mean free path for each beam velocity (dashed colored lines) and the physical length of a $L=500 \delta_{i}$ experiment at several densities (black lines) are overplotted for comparison.

\section{THE DESIGN SPACE FOR LABORATORY QUASI-PARALLEL SHOCK EXPERIMENTS}

Creating a quasi-parallel shock in a laboratory is a challenging endeavor. Simulations provide some guidance as to the parameters required ${ }^{18}$. A super-Alfvénic beam plasma must overlap a 5-10 times denser core plasma for the 2001000 ion-inertial lengths $\left(\delta_{i}=c / \omega_{p i}\right.$ where $\omega_{p i}$ is the ion plasma frequency) necessary for the shock to form. The core plasma density must be high enough to fit this many ioninertial lengths in the experimental apparatus, but low enough that Coulomb collisions remain negligible over that same distance. The background magnetic field must be strong enough to magnetize both plasmas over the experimental time scale but low enough that the beam satisfies the Mach number requirement. The usual magnetization criterion, $v_{b i, c i} \ll f_{c i}$ where $v_{b i, c i}$ is the beam ion/core ion Coulomb collision frequency and $f_{c i}$ is the ion cyclotron frequency, is here a necessary but insufficient condition. Beam instability growth and subsequent quasi-parallel shock formation require many ion gyroperiods $\left(f_{c i}^{-1}\right)$, leading to the usually more stringent condition $v_{b} / L \ll f_{c i}$. Studying these processes also requires that the ion gyroperiod and ion-inertial length must be wellresolvable by diagnostics. These competing conditions have yet to be simultaneously realized in an experiment.

Consider a laboratory experiment constituting a magnetized proton core plasma at rest in the lab frame and a less dense proton beam plasma streaming parallel to the ambient field. Assuming a practical system length upper limit of $L=10^{3}$ $\mathrm{cm}$ for a laboratory experiment implies a minimum practical core density of $n_{c} \geq 10^{14} \mathrm{~cm}^{-3}$ to observe the early stages of parallel shock formation $\left(\sim 500 \delta_{i}\right)$. At this density the core plasma will be collisional. However, for a beam velocity of $v_{b} \geq 10^{7} \mathrm{~cm} / \mathrm{s}$, the beam ion/core ion collisional mean free path $\lambda_{b i, c i}$ is much larger than $L$. This is possible because ${ }^{19}$ $\lambda_{b i, c i} \propto\left|v_{b}-v_{c}\right|^{4}$. Satisfying the condition that $M_{A} \geq 10$ for this beam velocity and core density requires a background magnetic field of $B_{0} \leq 100 \mathrm{G}$ (Fig. 1).

A similar experiment could be conducted at smaller length scales and higher densities: an $L=1 \mathrm{~cm}$ experiment would require a core density of $10^{20} \mathrm{~cm}^{-3}$. In order to maintiain $L<\lambda_{b i, c i}$, such an experiment would require $v_{b} \sim 10^{7} \mathrm{~cm} / \mathrm{s}$. Maintaining $M_{A}=10$ at this beam velocity would require a background field of $B_{0} \sim 1-10 \mathrm{~T}$ (Fig. 1).

The ideal beam plasma for a laboratory quasi-parallel shock would be a spatial and temporally uniform ion beam with a density $n_{b} \sim 0.01-0.1 n_{c}$ with no magnetic structure comparable to the ambient magnetic field strength. Electrostatic ion accelerators provide a uniform beam, but producing an ion beam with $1 \%$ of even the lowest core densities discussed above with such an accelerator is challenging. Bursts of plasma such as spheromaks ${ }^{20}$ and field-reversed configurations ${ }^{21}$ can be sufficiently dense and fast, but have substantial internal magnetic fields that may perturb the quasi-parallel field geometry. Laser-produced plasmas (LPPs) can be produced with densities and velocities in the desired range ${ }^{22}$. Significant magnetic fields are created near the target ${ }^{23}$, but most of the fast LPP ions escape this region into the relatively unperturbed ambient magnetic field. These characteristics make LPPs attractive drivers for laboratory quasi-parallel collisionless shock experiments.

\section{BEAM INSTABILITY THEORETICAL CONSIDERATIONS}

Two beam instabilities have been identified as important to coupling in quasi-parallel shocks: the right-hand resonant instability (RHI) and the non-resonant instability (NRI) . The right-hand resonant instability (RHI) is a gyroresonance between beam ions and EM waves near the ion cyclotron frequency on the whistler branch ${ }^{25}$. The NRI is also a gyroresonance (despite the misnomer), in this case between the core ions and similar (but somewhat lower frequency) EM waves $^{26}$. At high beam velocities and densities a higher frequency mode of each instability appears ${ }^{25}$.

When viewed in the rest frames of the beam and core ions respectively, the RHI and NRI both produce left-hand circularly polarized waves defined with respect to the ambient magnetic field direction. This polarization is consistent with ion cyclotron resonance. However, Doppler shifting to the laboratory/core-ion rest frame reverses the perceived polarization of the RHI to be right-handed. Careful consideration of the reference frame is therefore necessary when using the polarization to identify the instability responsible for 


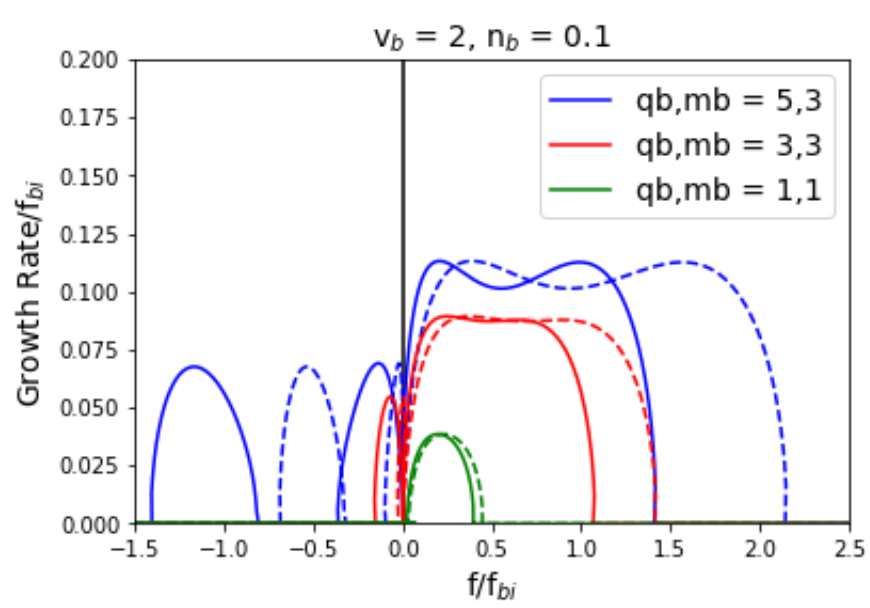

FIG. 2. Analytical instability growth rates in the electron rest (solid) and laboratory (dashed) frames for a variety of different beam/core ion combinations. The growth rates and frequencies are scaled to the beam ion cyclotron frequency $f_{b i}$. Peaks at positive and negative frequencies correspond to the RHI and NRI respectively. Increased charge and mass of beam ions can substantially increase the frequencies observed, especially in the laboratory frame. The frequencies generated do not simply scale with the charge/mass ratio.

an observed wave. Typically both spacecraft and laboratory probes are effectively stationary compared to the group velocity of the waves, so both make measurements in the laboratory frame. The polarization of waves measured in laboratory experiments is therefore directly comparable to spacecraft results.

In space, both beam and core ions are typically protons, and the corresponding frequencies observed are fractions of the ion cyclotron frequency. In laboratory experiments with higher atomic masses and charge states, however, waves are observed above the core ion cyclotron frequency ${ }^{16}$. These observations are consistent with analytical theory. The 1D cold linear dispersion relation for a beam ("b"), a core ("c") and a shared electron ("e") plasma in the electron rest frame (electron velocity $v_{e}=0$ ) can be written ${ }^{16,25}$

$$
\begin{aligned}
0 & =\omega^{2}-k^{2} c^{2} \\
& -\omega_{p c}^{2}\left(\frac{\omega-k v_{c}}{\omega-k v_{c}+\Omega_{c}}\right. \\
& +\frac{n_{b} q_{b}^{2}}{m_{b}} \frac{\omega-k v_{b}}{\omega-k v_{b}+\left(q_{b} / m_{b}\right) \Omega_{c}} \\
& \left.+\frac{n_{e} q_{e}^{2}}{m_{e}} \frac{\omega}{\omega+\left(q_{e} / m_{e}\right) \Omega_{c}}\right)
\end{aligned}
$$

where $q, n$, and $m$ are normalized to the core plasmas values and $\omega=\omega_{r}+i \gamma$ with real frequency $\omega_{r}$ and growth rate $\gamma$. Both the wave frequency and the growth rate depend on the ratio of the charge, mass, and density of the beam ions to the corresponding core ion quantities. These ratios cannot be factored into $\omega$, so no simple scaling of the frequency as a function of these parameters is possible. Figure 2 shows that even cases with equal charge/mass ratios have different

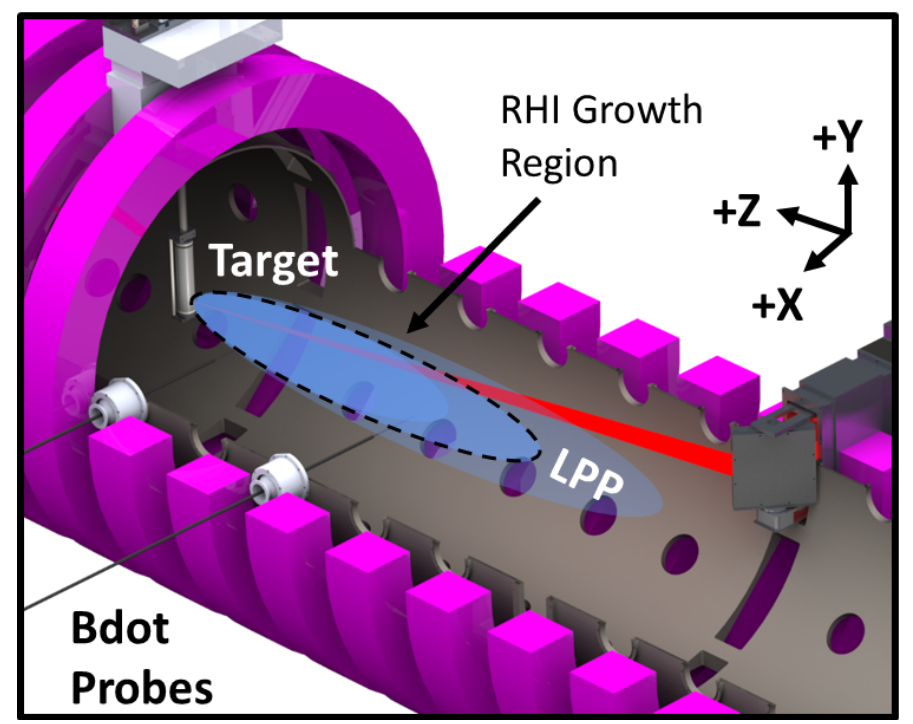

FIG. 3. Diagram of the experimental setup in the region near to the laser target. A black dashed line shows the approximate extent of the region in which beam instabilities grow in the current experiments.

solutions, which is a consequence of the $q_{b}^{2} / m_{b}$ term, and that the dependence of the frequency on these parameters is even more pronounced when waves are measured in the laboratory frame. Assuming the plasma is current-free, the relative velocity of the lab frame with respect to the electron reference frame is

$$
v_{e}=q_{b} n_{b} v_{b}
$$

so frequencies Doppler shifted from the electron rest frame to the laboratory frame include a further dependence on the charge, density and velocity of the ions. Laboratory experiments with heavier, more highly charged ions than protons can therefore produce considerably higher frequencies than those observed in space. Depending on the time scales of the experiment and frequency sensitivity of available diagnostics, this effect may be disadvantageous or beneficial.

\section{RESONANT INSTABILITY OBSERVATIONS}

A series of experiments have been conducted at UCLA to evaluate the potential of high-energy lasers as drivers of quasiparallel collisionless shocks in the type of large scale, low density experiment described in Section II. A pre-magnetized $18 \mathrm{~m}$ long, $30 \mathrm{~cm}$ diameter cylindrical helium core plasma is produced by two cathodes at either end of the Large Plasma Device (LAPD) ${ }^{15}$. The ion and electron temperatures are approximately $1 \mathrm{eV}$ and $5 \mathrm{eV}$ respectively. The plasma reaches densities of $\sim 10^{13} \mathrm{~cm}^{-3}$ at a minimum magnetic field of $300 \mathrm{G}$, corresponding to an Alfvén velocity of $10^{7} \mathrm{~cm} / \mathrm{s}$, an ion cyclotron frequency of $0.1 \mathrm{MHz}$, and a length of $L=80$ $\delta_{i}$. Simulations show this length to be sufficient for studying the growth of beam instabilities in the early stages of shock formation, but too short to fully form a shock ${ }^{18}$. 

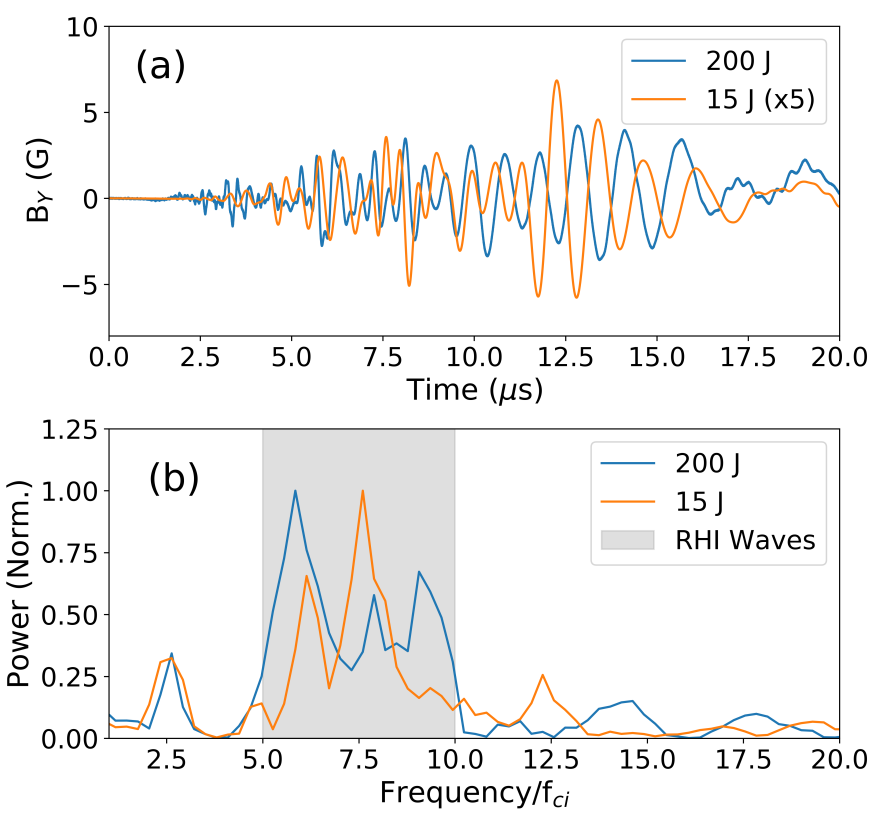

FIG. 4. a) Examples of waves driven by each laser observed $7.5 \mathrm{~m}$ from the laser target. The wave frequencies and relative amplitudes match, although the overall amplitude of the waves driven by the 15 $\mathrm{J}$ laser is $5 \mathrm{x}$ lower. b) A FFT of both wave traces shows that both have energy in a similar band. This frequency range is consistent with linear theory predictions for the RHI.

A high-energy laser is focused onto a plastic (high-density polyethelene, $\mathrm{C}_{2} \mathrm{H}_{4}$ ) target embedded in the LAPD plasma, and the resulting laser-produced plasma (LPP) "beam" streams anti-parallel to the background magnetic field (Fig. 3). The target is positioned near one end of the LAPD, leaving a $\sim 14 \mathrm{~m}$ long experimental volume. Two lasers are used in separate experiments. The Raptor laser ${ }^{30}(1053 \mathrm{~nm}, 25 \mathrm{~ns}, 200 \mathrm{~J}$, $1 \mathrm{shot} / \mathrm{hr}$ ) is used to drive beam instabilities to the largest possible amplitudes, while the Peening $\operatorname{laser}^{31}$ (1053 nm, $15 \mathrm{ns,}$ $15 \mathrm{~J}, 1 \mathrm{shot} / \mathrm{s}$ ) is used to collect volumetric and high-statistic datasets at lower wave amplitudes. Both lasers are focused to a maximum intensity of $\sim 10^{13} \mathrm{~W} / \mathrm{cm}^{2}$ to generate a carbon LPP with charge stages from $\mathrm{C}^{+2}$ to $\mathrm{C}^{+5}$ and velocities ranging from $1-5 \times 10^{7} \mathrm{~cm} / \mathrm{s}\left(M_{A}=1-5\right)^{16}$. Fast protons are also produced, but similar results with graphite targets suggest that the carbon ions are dominant in these experiments. The beam ion/core ion Coulomb mean free path is $>1 \mathrm{~km}$, so the ion/ion interaction is effectively collisionless. The laser spot on target defines the spatial origin of a right-handed coordinate system with the background magnetic field in the $+Z$ and the target aligned along $+Y$. The arrival of the laser on target defines $t=0$.

Electromagnetic waves generated by beam instabilities in the experiment are measured by an array of 3-axis, $3 \mathrm{~mm}$ diameter magnetic flux probes ${ }^{32}$. The voltages induced in the probes are differentially amplified, digitized at $1.25 \mathrm{GHz}$, then numerically integrated to calculated $\Delta B$. The probes are positioned by motorized probe drives to enable fully autonomous collection of large volumetric datasets. Waves are observed in
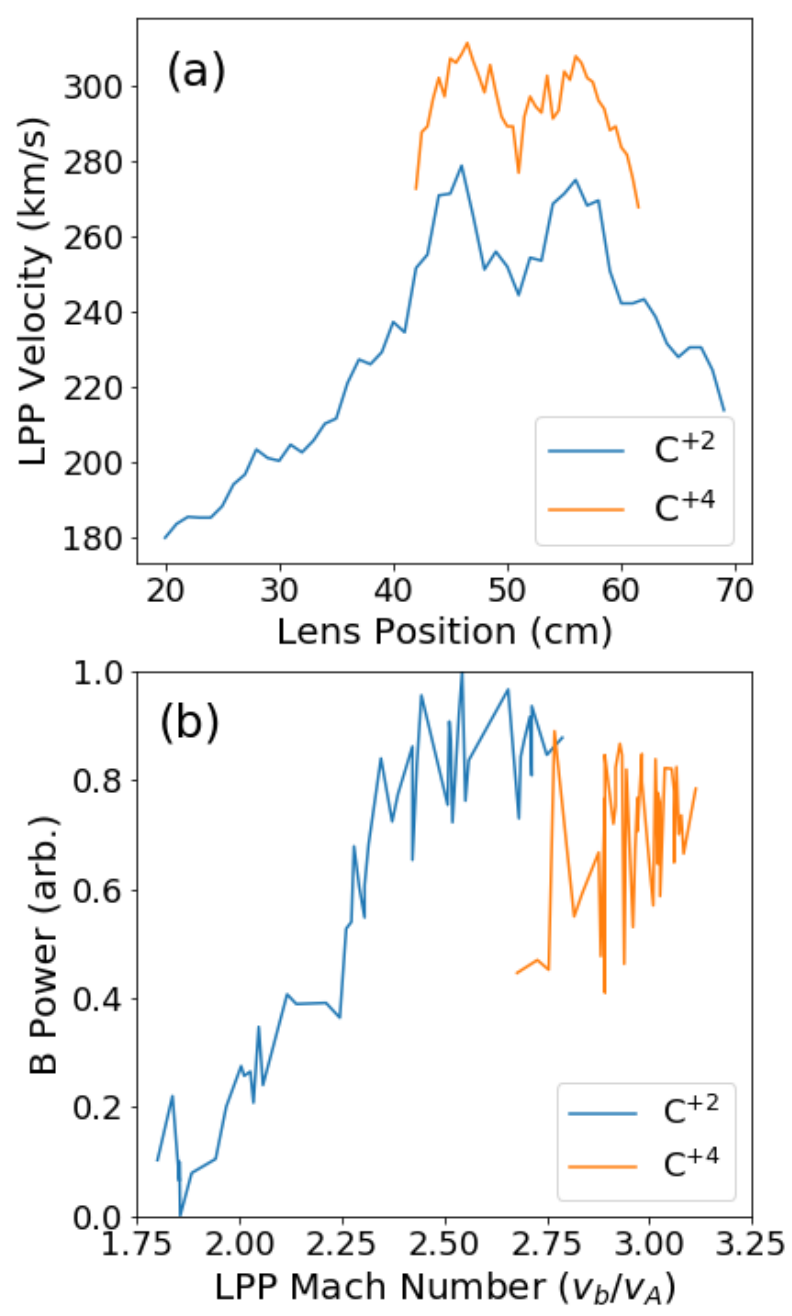

FIG. 5. a) Leading-edge velocities of $\mathrm{C}^{+2}$ and $\mathrm{C}^{+4}$ measured using the high repetition rate Peening laser as the final laser focusing lens was translated to vary the intensity on target. Two peaks in velocity reflect two separate laser focii due to the angled final focusing lens. The $\mathrm{C}^{+4}$ scan covers a smaller range of lens positions because negligible $\mathrm{C}^{+4}$ is created at lower intensities. b) Maximum magnetic field amplitude in the RHI frequency range over the same datasets, sorted by and plotted against the measured LPP velocity. The wave power increases linearly with laser intensity, as does the velocity of each charge state.

experiments with both lasers. The waves observed are righthand circularly polarized, consistent with generation by the $\mathrm{RHI}^{16}$. Each velocity and charge state in the LPP corresponds to a different Doppler-shifted cyclotron resonance, so a range of wave frequencies from 2-15 $f_{c i}$ is observed, with most of the spectral energy concentrated between 5-10 $f_{c i}$ (Fig. 4b). These frequencies are normalized to the core ion cyclotron frequency to avoid confusion between the multiple beam ion species. 


\section{A. Dependence of Wave Amplitude on Beam Velocity}

Linear theory ${ }^{16,25}$ shows that the growth rate of the RHI depends strongly on the velocity and density of the beam ions relative to the core. These parameters can be varied experimentally by changing the intensity of the laser on target while maintaining constant energy. To study this dependence, the wave amplitude at a fixed location and frequency is measured while the focal spot size is systematically varied by translating the final focusing lens. At the same time, the velocity distribution of a single beam ion species is determined for each shot by time-of-flight (assuming the LPP starts at the target at $t=0$ ) using time-resolved monochromator measurements of ion fluorescence. The focusing lens is intentionally angled (to control lens ghosts), introducing some astigmatism. As the lens translates, the intensity therefore passes through two maxima corresponding to separate horizontal and vertical foci (Fig. 5a). Each lens position is an average over ten shots.

For each velocity distribution, a characteristic maximum velocity is defined by the leading edge at $25 \%$ rise. Fig. 5a shows the velocity of two species of Carbon as a function of lens position, clearly showing the two separate foci. The velocities of both species have a similar dependence on intensity, though the higher charge state is always faster. The $\mathrm{C}^{+4}$ scan covers a smaller range of lens positions because negligible $\mathrm{C}^{+4}$ is created at lower intensities. This result is consistent with LPP characterization experiments ${ }^{22}$.

The waves generated during the same shots are measured by a magnetic flux probe. Fig. 5b shows the maximum wave amplitude in one of the RHI frequency bands $\left(3.5-5.5 f_{c i}\right)$ sorted by and plotted against the corresponding measured leading-edge LPP velocities. In this regime the maximum RHI amplitude is proportional to $v_{b}$. For the velocity ranges measured this scaling is consistent with the growth rate predicted by solving the linear dispersion relation (Eq 1). The LPP density in the growth region was not measured, but linear theory predicts a relatively weak dependence on density ${ }^{16}$ at low Mach number and densities $\geq 5 \%$.

\section{B. Localizing the RHI Growth Region}

Previous work suggested that the growth of beam instabilities was confined to a small region near the target ${ }^{16}$. This hypothesis is confirmed by direct measurement of the wave amplitude at one of the RHI frequency peaks $\left(\mathrm{f} \sim 5 \mathrm{f}_{c i}\right)$ at increasing distances from the target (Fig. 6). An array of magnetic flux probes provides measurements far from the target, while a single probe is moved between shots to obtain higher spatial resolution near the target. Shot-to-shot errors are quantified by repeating five shots at a single set of positions.

The measured wave amplitude begins low, then increases quickly between $25-50 \mathrm{~cm}\left(2-5 \delta_{c i}\right)$ from the target. After this region the wave amplitude falls off as approximately $1 / z^{2}$ as the waves spatially disperse. The RHI will be nominally stabilized when either all the free energy from sufficiently fast ions has been depleted or when the LPP density drops far below the background density (in the latter case the growth rate

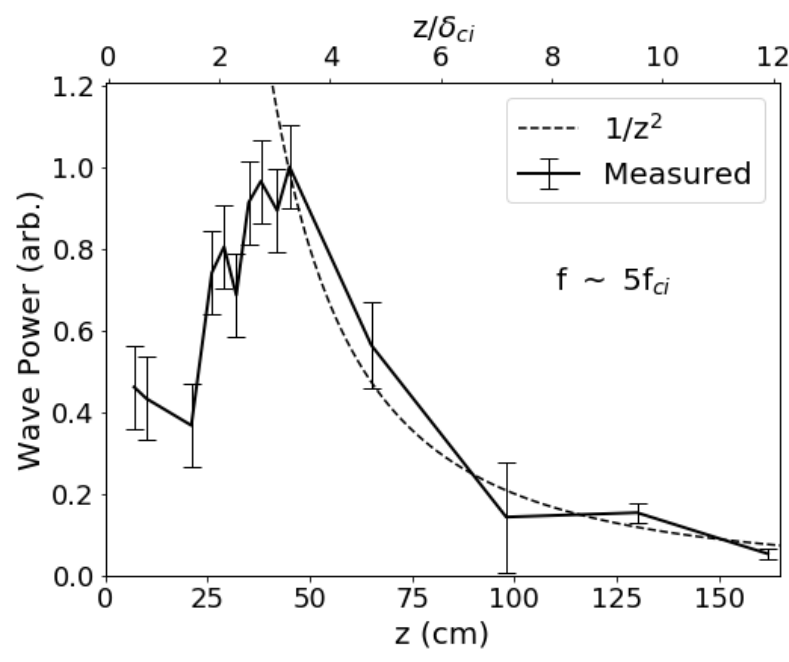

FIG. 6. Spatially-resolved measurements of the maximum wave amplitude achieved near one of the RHI frequency peaks show that growth of the RHI is constrained to a region of $\sim 50 \mathrm{~cm}$ near the laser target. After this region, the wave amplitude decays as approximately $1 / z^{2}$.

becomes negligible but remains non-zero). In this experiment, super-Alfvénic ions are still observed far from the laser target $^{16}$, indicating that the LPP density is the factor limiting wave growth.

\section{MODELING LPP DENSITY DISPERSION}

The model chosen must retain ion cyclotron motion (which is a dominant feature over the time scales of interest), so the fluid approximation is not appropriate. At the same time, the model must simulate the entire large experimental volume in three dimensions, making particle-in-cell or hybrid simulations prohibitively expensive. The problem can be greatly simplified by assuming that interactions between LPP ions as well as interactions among LPP ions and core ions can be neglected. Under this assumption, ions follow single particle trajectories that are easily solvable analytically. The evolution of the LPP density can then be estimated by a Monte-Carlo calculation.

A distribution of $\geq 10^{5}$ quasi-particles is initiated with velocity vector and charge state distributions consistent with experimental measurements ${ }^{33}$. The helical trajectories of each particle are then calculated analytically using the cyclotron equations

$$
\begin{aligned}
& x(t)=r_{L} \cos \left(\omega_{c i} t+\psi\right)+x_{G C} \\
& y(t)=r_{L} \sin \left(\omega_{c i} t+\psi\right)+y_{G C} \\
& z(t)=v_{z} t
\end{aligned}
$$

where $r_{L}, \psi$, and $v_{z}$ are the Larmor radius, gyrophase, and parallel velocity and $\left(x_{G C}, y_{G C}\right)$ is the center of gyration. At each time-step a random-walk is applied to each particle in the transverse plane to model cross-field diffusion. The random 

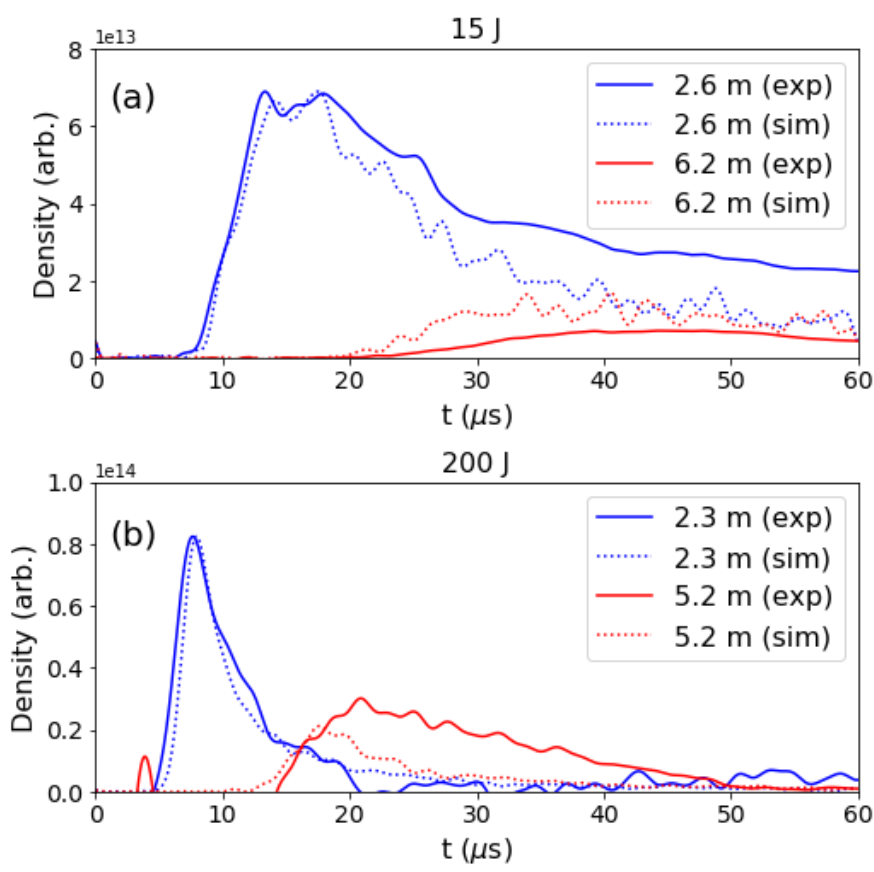

FIG. 7. A comparison of Langmuir probe ion saturation current measurements and "virtual" Langmuir probe measurements from MonteCarlo calculations is used to adjust the initial particle velocity distribution and cross-field diffusion coefficient. This comparison is shown for the $15 \mathrm{~J}$ (a) and $200 \mathrm{~J}$ (b) lasers.

walk step size is

$$
\delta_{D}=\sqrt{D d t}
$$

where $D$ is the diffusion coefficient. The diffusion coefficient is determined to be $D \approx 5000 \mathrm{~m}^{2} / \mathrm{s}$ by comparing calculation results to Langmuir probe density measurements from several positions in the experiment (Fig. 7). Along with , this cross-field transport explains the discrepancies between Monte-Carlo calculations and measurements noted in our previous work $^{16}$.

The quasi-particle density at a given space and time is calculated by counting the number of quasi-particles in a defined volume. This density is then converted to an approximate actual plasma density by assuming the total number of ablated LPP particles to be $N_{p} \approx 5 \times 10^{16}$, consistent with previous experimental measurements ${ }^{22}$. Fig. 8a shows the calculated density evolution at $(\mathrm{x}, \mathrm{y})=(0,0)$ for a $200 \mathrm{~J}$ laser shot. The approximate RHI growth region where $n_{b} / n_{c}>4 \%$ (bounded by a black contour) is chosen to be consistent with Fig. 6 .

A possible scheme for maintaining the LPP uniformity and density over longer distances is the use of pulse shaping to produce a train of laser pulses separated in time ${ }^{34,35}$. If the pulses are spaced sufficiently close together, their velocity distributions will cause them to merge together to form a quasicontinuous LPP. In addition to extending the LPP in space, spreading energy output in time also allows the total pulse energy to be increased without increasing the maximum peak intensity. Since the maximum laser energy is limited by the
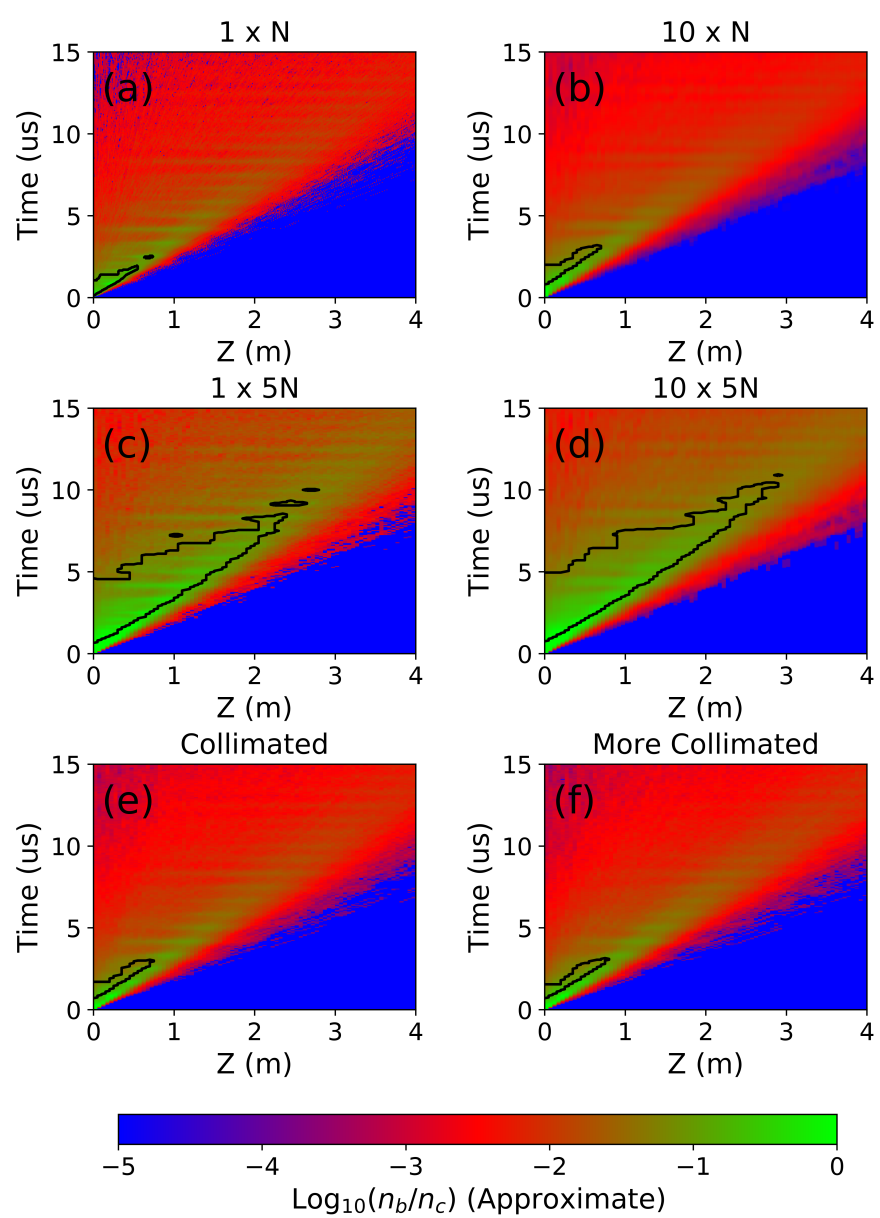

FIG. 8. Calculated density distributions displayed as a function of distance from the laser target and time with a contour (black) marking the edge of the instability growth region where $n_{b} / n_{c} \geq 4 \%$. Six cases are shown: a) $N_{p}$ particles in a single pulse b) $N_{p}$ particles distributed between ten pulses each separated by $100 \mathrm{~ns}$ c) $5 N_{p}$ particles in a single pulse d) $5 N_{p}$ particles in ten pulses separated by $100 \mathrm{~ns}$ e) A single pulse of $N_{p}$ particles with a more collimated angular velocity distribution $v_{b} \propto \cos ^{4}(\theta)$ and f) A single pulse of $N_{p}$ particles with $v_{b} \propto \cos ^{8}(\theta)$. Adding multiple pulses slightly increases the extent of the high density region, but increasing the number of particles has a much larger effect. A more collimated velocity distribution also slightly extends the instability growth region.

intensity-based damage thresholds of its optical components, a train of pulses allows the laser to deposit a higher total energy into the target. Using a series of short pulses rather than a continuous long pulse maintains high instantaneous intensity and therefore keeps LPP velocities comparable to those achieved in the single-pulse experiments

Preliminary Monte-Carlo calculations suggest that, for the LPP velocity distributions measured experimentally, a pulse separation on the order of $100 \mathrm{~ns}$ will best approximate a quasi-continuous LPP. The ideal pulse separation depends on the laser parameters, as more narrow velocity distributions require tighter pulse spacing in order for pulses to merge. Taking the number of particles in a single pulse $\left(N_{p}=5 \times 10^{16}\right)$ 
and dividing it between ten pulses provides a modest improvement in the growth region (Fig. 8b). However, providing a train of 10 pulses with a total of $5 N_{p}$ particles leads to a substantially extended growth region (Fig. 8d). A single pulse of $5 N_{p}$ (Fig. 8c) also has an enhanced growth region, although it remains somewhat shorter than the ten pulse case. This model predicts that a series of sufficiently closely-spaced pulses will merge to produce a long, relatively homogeneous extended LPP ideal for beam instability growth.

LPP density uniformity could also be improved by producing a more collimated LPP, possibly by using a shaped target. The LPP velocity distribution is assumed to be proportional to $\cos ^{n}(\theta)$ where $\theta$ is the angle to the target normal. Experiments have shown that $n \approx 2$ for a flat-surface $\operatorname{target}^{33}$. Figures $8 \mathrm{e}$ and $8 \mathrm{f}$ are identical to Fig. 8a except that they assume more narrow angular velocity distributions with $n=4$ and $n=8$ respectively. Some improvement is evident at $n=4$ but little further improvement is found for $n>4$ as LPP divergence eventually becomes negligible compared to cross-field diffusion and parallel velocity dispersion.

\section{CONCLUSIONS}

Quasi-parallel collisionless shocks are objects of considerable interest, and generating one in a well-diagnosed laboratory environment would improve our understanding of their formation and structure. However, scaling a quasi-parallel shock to a feasible experiment is a challenge. The choice of experiment length scale and shock driver dictate demanding requirements on the required background density and magnetic field. Laser-produced plasmas (LPPs) provide a promising driver for such experiments.

A series of experiments at UCLA has investigated LPPs in this context and observed electromagnetic ion/ion beam instability growth consistent with the very early stages of quasiparallel shock formation. However spatial dispersion of the LPP prematurely terminates the growth of the instability. This limitation is both demonstrated directly using magnetic flux probe measurements and reproduced by a Monte-Carlo calculation. This Monte-Carlo model is also used to evaluate the use of trains of laser pulses to partially mitigate LPP dispersion with promising results. Future experiments and hybrid simulations will pursue this approach to develop an improved LPP quasi-parallel shock driver.

\section{ACKNOWLEDGMENTS}

This work was supported by the Defense Threat Reduction Agency, Lawrence Livermore National Security LLC, and the United States Department of Energy (DOE) under contract No. DE-SC0017900. The Peening laser was made available by the Naval Information Warfare Center Pacific under contract No. NCRADA-NIWC Pacific-19-354. M.S.W. was partially supported by the Deutsche Forschungsgemeinschaft. The experiments were performed at the UCLA Basic Plasma Science Facility (BaPSF), which is a collaborative research facility supported by the U. S. Department of Energy, Office of Science, Office of Fusion Energy Sciences, and the National Science Foundation. We would like to thank the staff of BaPSF, Z. Lucky, M. Drandell, T. Ly, and A. Kohli for their help conducting the experiments.

${ }^{1}$ R. D. Blandford and J. P. Ostriker, The Astrophysical Journal 221, L29 (1978).

${ }^{2}$ A. R. Bell, Monthly Notices of the Royal Astronomical Society 182, 147 (1978).

${ }^{3}$ E. W. Greenstadt, in Collisionless Shocks in the Heliosphere: Reviews of Current Research (American Geophysical Union, 1985) pp. 169-184.

${ }^{4}$ F. Fiuza, R. A. Fonseca, J. Tonge, W. B. Mori, and L. O. Silva, Physical Review Letters 108 (2012), 10.1103/physrevlett.108.235004.

${ }^{5}$ D. Winske and M. M. Leroy, Journal of Geophysical Research 89, 2673 (1984).

${ }^{6}$ D. Burgess and M. Scholer, Space Science Reviews (2012).

${ }^{7}$ L. Gargaté and A. Spitkovsky, The Astrophysical Journal 744, 67 (2011).

${ }^{8}$ M. Ackermann, M. Ajello, A. Allafort, L. Baldini, J. Ballet, G. Barbiellini, M. G. Baring, D. Bastieri, K. Bechtol, R. Bellazzini, R. D. Blandford, E. D. Bloom, E. Bonamente, A. W. Borgland, E. Bottacini, T. J. Brandt, J. Bregeon, M. Brigida, P. Bruel, R. Buehler, G. Busetto, S. Buson, G. A. Caliandro, R. A. Cameron, P. A. Caraveo, J. M. Casandjian, C. Cecchi, Ö. Çelik, E. Charles, S. Chaty, R. C. G. Chaves, A. Chekhtman, C. C. Cheung, J. Chiang, G. Chiaro, A. N. Cillis, S. Ciprini, R. Claus, J. CohenTanugi, L. R. Cominsky, J. Conrad, S. Corbel, S. Cutini, F. D’Ammando, A. de Angelis, F. de Palma, C. D. Dermer, E. do Couto e Silva, P. S. Drell, A. Drlica-Wagner, L. Falletti, C. Favuzzi, E. C. Ferrara, A. Franckowiak, Y. Fukazawa, S. Funk, P. Fusco, F. Gargano, S. Germani, N. Giglietto, P. Giommi, F. Giordano, M. Giroletti, T. Glanzman, G. Godfrey, I. A. Grenier, M.-H. Grondin, J. E. Grove, S. Guiriec, D. Hadasch, Y. Hanabata, A. K. Harding, M. Hayashida, K. Hayashi, E. Hays, J. W. Hewitt, A. B. Hill, R. E. Hughes, M. S. Jackson, T. Jogler, G. Jóhannesson, A. S. Johnson, T. Kamae, J. Kataoka, J. Katsuta, J. Knödlseder, M. Kuss, J. Lande, S. Larsson, L. Latronico, M. Lemoine-Goumard, F. Longo, F. Loparco, M. N. Lovellette, P. Lubrano, G. M. Madejski, F. Massaro, M. Mayer, M. N. Mazziotta, J. E. McEnery, J. Mehault, P. F. Michelson, R. P. Mignani, W. Mitthumsiri, T. Mizuno, A. A. Moiseev, M. E. Monzani, A. Morselli, I. V. Moskalenko, S. Murgia, T. Nakamori, R. Nemmen, E. Nuss, M. Ohno, T. Ohsugi, N. Omodei, M. Orienti, E. Orlando, J. F. Ormes, D. Paneque, J. S. Perkins, M. Pesce-Rollins, F. Piron, G. Pivato, S. Rainò, R. Rando, M. Razzano, S. Razzaque, A. Reimer, O. Reimer, S. Ritz, C. Romoli, M. Sánchez-Conde, A. Schulz, C. Sgrò, P. E. Simeon, E. J. Siskind, D. A. Smith, G. Spandre, P. Spinelli, F. W. Stecker, A. W. Strong, D. J. Suson, H. Tajima, H. Takahashi, T. Takahashi, T. Tanaka, J. G. Thayer, J. B. Thayer, D. J. Thompson, S. E. Thorsett, L. Tibaldo, O. Tibolla, M. Tinivella, E. Troja, Y. Uchiyama, T. L. Usher, J. Vandenbroucke, V. Vasileiou, G. Vianello, V. Vitale, A. P. Waite, M. Werner, B. L. Winer, K. S. Wood, M. Wood, R. Yamazaki, Z. Yang, and S. Zimmer, Science 339, 807 (2013), http://science.sciencemag.org/content/339/6121/807.full.pdf.

${ }^{9}$ F. L. Scarf, D. A. Gurnett, W. S. Kurth, and R. L. Poynter, Nature 280, 796 (1979).

${ }^{10}$ L. B. Wilson, C. Cattell, P. J. Kellogg, K. Goetz, K. Kersten, L. Hanson, R. MacGregor, and J. C. Kasper, Physical Review Letters 99 (2007), 10.1103/physrevlett.99.041101.

${ }^{11}$ E. A. Lucek, T. S. Horbury, I. Dandouras, and H. Rème, Journal of Geophysical Research: Space Physics 113, n/a (2008).

${ }^{12}$ R. P. Drake, Physics of Plasmas 7, 4690 (2000).

${ }^{13}$ C. Niemann, W. Gekelman, C. G. Constantin, E. T. Everson, D. B. Schaeffer, A. S. Bondarenko, S. E. Clark, D. Winske, S. Vincena, B. V. Compernolle, and P. Pribyl, Geophysical Research Letters 41, 7413 (2014).

${ }^{14}$ D. B. Schaeffer, W. Fox, D. Haberberger, G. Fiksel, A. Bhattacharjee, D. H. Barnak, S. X. Hu, and K. Germaschewski, Phys. Rev. Lett. 119 (2017).

${ }^{15}$ W. Gekelman, P. Pribyl, Z. Lucky, M. Drandell, D. Leneman, J. Maggs, S. Vincena, B. Van Compernolle, S. K. P. Tripathi, G. Morales, T. A. Carter, Y. Wang, and T. DeHaas, Review of Scientific Instruments 87 (2016), https://doi.org/10.1063/1.4941079.

${ }^{16}$ P. V. Heuer, M. S. Weidl, R. S. Dorst, D. B. Schaeffer, A. S. Bondarenko, S. K. P. Tripathi, B. Van Compernolle, S. Vincena, C. G. Constantin, 
C. Niemann, and D. Winske, Physics of Plasmas 25, 032104 (2018), https://doi.org/10.1063/1.5017637.

${ }^{17}$ P. V. Heuer, M. S. Weidl, R. S. Dorst, D. B. Schaeffer, S. K. P. Tripathi, S. Vincena, C. G. Constantin, C. Niemann, L. B. Wilson, and D. Winske, The Astrophysical Journal Letters (2020), (in press).

${ }^{18}$ M. S. Weidl, D. Winske, F. Jenko, and C. Niemann, Physics of Plasmas 23 (2016), https://doi.org/10.1063/1.4971231.

${ }^{19}$ D. W. Koopman, Physics of Fluids 15, 1959 (1972).

${ }^{20}$ T. R. Jarboe, Plasma Physics and Controlled Fusion 36, 945 (1994)

${ }^{21}$ L. C. Steinhauer, Physics of Plasmas 18, 070501 (2011).

${ }^{22}$ D. B. Schaeffer, A. S. Bondarenko, E. T. Everson, S. E. Clark, C. G. Constantin, and C. Niemann, Journal of Applied Physics 120, 043301 (2016).

${ }^{23}$ A. Collette and W. Gekelman, Physical Review Letters 105 (2010), 10.1103/physrevlett.105.195003.

${ }^{24}$ S. P. Gary, Space Science Reviews 56, 373 (1991).

${ }^{25}$ M. S. Weidl, D. Winske, and C. Niemann, The Astrophysical Journal 873, 57 (2019).

${ }^{26}$ M. S. Weidl, D. Winske, and C. Niemann, The Astrophysical Journal 872, 48 (2019)

${ }^{27}$ K. B. Quest, Journal of Geophysical Research: Space Physics (1978-2012) 93, 9649 (1988).
${ }^{28}$ N. Omidi, H. Karimabadi, D. Krauss-Varban, and K. Killen, "Generation and nonlinear evolution of oblique magnetosonic waves: Application to foreshock and comets," in Solar System Plasmas in Space and Time (American Geophysical Union, 1994) pp. 71-84.

${ }^{29}$ K. Killen, N. Omidi, D. Krauss-Varban, and H. Karimabadi, Journal of Geophysical Research 100, 5835 (1995).

${ }^{30}$ C. Niemann, C. G. Constantin, D. B. Schaeffer, A. Tauschwitz, T. Weiland, Z. Lucky, W. Gekelman, E. T. Everson, and D. Winske, Journal of Instrumentation 7, P03010 (2012).

${ }^{31}$ L. Hackel, J. Miller, and C. Dane, International Journal of Nonlinear Optical Physics 2, 171 (1993).

${ }^{32}$ E. T. Everson, P. Pribyl, C. G. Constantin, A. Zylstra, D. Schaeffer, N. L. Kugland, and C. Niemann, Rev. Sci. Insturm. 80, 113505 (2009).

${ }^{33}$ P. Heuer, D. Schaeffer, E. Knall, C. Constantin, L. Hofer, S. Vincena, S. Tripathi, and C. Niemann, High Energy Density Physics 22, 17 (2017).

${ }^{34}$ A. G. Berezutsky, V. N. Tishchenko, Y. P. Zakharov, I. B. Miroshnichenko, and I. F. Shaikhislamov, Quantum Electronics 49, 178 (2019).

${ }^{35}$ V. N. Tishchenko, A. G. Berezutsky, E. L. Boyarintsev, Y. P. Zakharov, I. B. Miroshnichenko, V. G. Posukh, A. G. Ponomarenko, A. A. Chibranov, and I. F. Shaikhislamov, Journal of Physics: Conference Series 1404, 012100 (2019). 